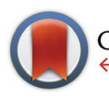

CrossMark \&lick for updates

Cite this: Polym. Chem., 2017, 8, 936

\title{
Amphiphilic and double hydrophilic block copolymers containing a polydehydroalanine block $\uparrow$
}

\author{
Mark Billing, ${ }^{a, b}$ Grit Festag, ${ }^{a, b}$ Peter Bellstedt ${ }^{a}$ and Felix H. Schacher ${ }^{\star a, b}$
}

We present the synthesis and characterization of amphiphilic and double hydrophilic block copolymers containing a polydehydroalanine (PDha) block. First, polystyrene-block-poly(tert-butoxycarbonylaminomethyl acrylate) (PS-b-PtBAMA) and poly(n-butylacrylate)-block-poly(tert-butoxycarbonylaminomethyl acrylate) (PnBA-b-PtBAMA) were prepared by atom transfer radical polymerization (ATRP). Subsequent deprotection led to the corresponding PS- $b$-PDha and PnBA-b-PDha block copolymers. All intermediate stages were characterized by ${ }^{1} \mathrm{H}-,{ }^{13} \mathrm{C}$ - or solid state MAS ${ }^{13} \mathrm{C}-\mathrm{NMR}$. Afterwards, the solution behavior of PAA- $b$-PDha was investigated by a combination of dynamic light scattering, zeta potential measurements, and potentiometric titration - revealing that aggregation occurs upon reaching $\mathrm{pH}$ values of 3 or lower. In case of PS- $b$-PDha, core-corona micelles are formed in aqueous solution, as revealed by a combination of DLS and cryogenic transmission electron microscopy.

Received 29th November 2016
Accepted 21st December 2016

DOI: $10.1039 /$ c6py02076c

rsc.li/polymers

\section{Introduction}

Polymers carrying charged groups are generally referred to as polyelectrolytes ${ }^{1,2}$ and, depending on the nature of the functional groups, can be further subdivided into strong and weak polycations and polyanions. ${ }^{3}$ Whereas strong polyelectrolytes remain permanently charged irrespective of the $\mathrm{pH}$ value of the surrounding medium, weak polyelectrolytes exhibit $\mathrm{pH}$ dependent charge characteristics. A further subclass, polyampholytes, features both cationic and anionic charges, either as copolymers or as charged segments in ampholytic block copolymers, ${ }^{4}$ whereas polyzwitterions are typically characterized by both charges being located within the same monomer unit. $^{3,5-7}$ All these materials attract continuing interest in different fields of science, as their solution behavior can be influenced through external stimuli such as ionic strength, changes in solvent quality, temperature, or nature and valency of the respective counterions. ${ }^{8-10}$ As a result, a wide range of applications has been reported for polyelectrolytes and related materials, including sewage treatment, as metal binders (e.g. Cd) in oil recovery, water purification, or as flocculants and emulsifiers. ${ }^{5}$ In case of polyzwitterions, specific appli-

\footnotetext{
${ }^{a}$ Laboratory of Organic Chemistry and Macromolecular Chemistry, Friedrich-Schiller-University Jena, Humboldtstraße 10, D-07743 Jena, Germany. E-mail:felix.schacher@uni-jena.de

${ }^{b}$ Jena Center for Soft Matter (JCSM), Friedrich-Schiller-University Jena, Philosophenweg 7, D-07743 Jena, Germany

$\dagger$ Electronic supplementary information (ESI) available. See DOI: 10.1039/ c6py02076c
}

cations in antifouling coatings ${ }^{11}$ or membranes ${ }^{12}$ are constantly discussed. Even their use as protective layers for sensors and membranes to extend their lifetime in medical applications or to improve their sensitivity has been reported. ${ }^{12,13}$

Examples for polyampholytes synthesized by free radical polymerization are copolymers of quaternized $\operatorname{poly}((N, N$-dimethylaminoethyl acrylate)-co-(sodium styrene sulfonic acid)) P(DMAEAq- $c o-N a S S){ }^{14}$ or systems composed of $\mathrm{N}$-(3-aminopropyl) methacrylamide hydrochloride and acrylic acid P(APMco-MAA). ${ }^{15}$ In another example, polyampholyte membranes composed of 2-acrylamide-2-methyl propane sulfonic acid (AMPS) and [2-(methacryloyloxy)ethyl] trimethylammonium chloride (DMC) were reported. ${ }^{16}$ Reversible deactivation radical polymerization (RDRP) techniques have also been used to synthesize well-defined polyampholytes, as shown for poly ( $N, N$-dimethylaminoethyl methacrylate) (PDMAEMA)/poly (methacrylic acid) (PMAA) block copolymer brushes using ARGET ATRP, ${ }^{17}$ or the copolymerization of DMAEMA and MAA $^{18}$ or 4 -vinylbenzoic acid and 4-vinylbenzyl(triphenylphosphonium) chloride via reversible addition fragmentation chain transfer polymerization (RAFT). ${ }^{19}$

Regarding block polyampholytes, first examples consisting of poly(2-vinylpyridine) (P2VP) and different acrylates/methacrylates were presented by Kamachi et al. ${ }^{20}$ Giebeler and Stadler later studied the aqueous solution behavior of polyampholytic triblock terpolymers containing both a PMAA and a P2VP segment. ${ }^{21}$ Such materials were afterwards used for the preparation of multicompartment micelles and, starting from that, the synthesis of nanoscopic hybrid materials, ${ }^{22,23}$ 
compartmentalized interpolyelectrolyte complexes, ${ }^{24}$ and as non-viral vectors in gene delivery applications. ${ }^{25}$ The temperature responsive self-assembly of zwitterionic block copolymers was recently investigated by the group of Yoshida. ${ }^{26}$ Here, block copolymers containing $N, N$-dimethyl- $N$-(3-(methacrylamido) propyl)aminopropanesulfonate and $N$-isopropylacrylamide were synthesized via RAFT polymerization and their self-assembly was investigated. In another example, zwitterionic poly(2-methoxyethyl acrylate)-block-poly(3-( $N$-(2-methacryloyloxyethyl)- $N, N$-dimethylamino)propane sulfonate) (PMEA- $b$-PDMAPS) block copolymers were synthesized using ATRP. ${ }^{27}$

In this context, we recently reported on tert-butoxycarbonylaminomethyl acrylate ( $t$ BAMA) as versatile building block for the preparation of polyampholytes and its polymerization using either free radical polymerization or atom transfer radical polymerization (ATRP). ${ }^{28,29}$ The use of (in principle) orthogonal protective groups for the amino function (tertbutoxy carbonyl) and the carboxylic acid (methyl ester) allows for selective deprotection of either the two functionalities. ${ }^{29}$ The formed polymers can be subsequently converted into weak polycations, polyanions, or zwitterionic polydehydroalanine (PDha) featuring high charge densities. ${ }^{29,30}$ Related to that, earlier attempts by Hermes and Mathias employed free radical polymerization of different capto dative methyl(2-alkanamido) propenoates. ${ }^{31-33}$

In this contribution, we report on the preparation and solution properties of block copolymers containing a polydehydroalanine block (PDha). Starting from previously synthesized PS- $b$-P $t$ BAMA and P $n$ BA- $b$-P $t$ BAMA block copolymers, different deprotection reactions led to amphiphilic or double hydrophilic block copolymers. Thereby, we discovered that our previous assumption that either the $-\mathrm{COOH}$ or $-\mathrm{NH}_{2}$ moiety of PtBAMA can be selectively deprotected has to be reconsidered. In both cases, considerable amounts of the other protective group (Boc in the case of $-\mathrm{NH}_{2}$ and methyl ester for $-\mathrm{COOH}$ ) is cleaved off as well. Nevertheless, we show first investigations of the solution properties of the resulting amphiphilic PS- $b$-PDha or double hydrophilic PAA- $b$-PDha block copolymers in aqueous media.

\section{Experimental section}

Trifluoroacetic acid (Carl Roth GmbH+Co. KG, $\geq 99.9 \%$ ) and lithiumhydroxide monohydrate (Carl Roth $\mathrm{GmbH}+\mathrm{Co} . \mathrm{Kg}$, $\geq 55 \%$ ) were used as received. $\mathrm{N}$-(tert-Butoxycarbonyl)-D-serine methyl ester (Carbolution, 98\%) was used as received. Styrene (Sigma Aldrich, $\geq 99 \%$ ) contained 4-tert-butylcatechol as inhibitor which was removed by column chromatography over neutral aluminum oxide and subsequent distillation before use. The inhibitor of $N$-butyl acrylate (Sigma Aldrich, $\geq 99.9 \%$ ) was removed by column chromatography over neutral aluminum oxide. Tetrahydrofuran (THF) and dichloromethane (DCM) were purified using a PureSolv-ENTM Solvent purification System (Innovative Technology). Any glassware was cleaned in a $\mathrm{KOH} /$ iso-propanol bath and dried at $110{ }^{\circ} \mathrm{C}$. All deuterated solvents were obtained from Deutero. For dialysis, a Spectra/Por®Dialysis membrane with a nominal molecular weight cut-off (MWCO) of $1000 \mathrm{~g} \mathrm{~mol} \mathrm{~m}^{-1}$ (RC) was used.

\section{Nuclear magnetic resonance spectroscopy (NMR)}

${ }^{1} \mathrm{H}-\mathrm{NMR}$ and ${ }^{13} \mathrm{C}-\mathrm{NMR}$ spectra were recorded in $\mathrm{CDCl}_{3}$, $\mathrm{CD}_{2} \mathrm{Cl}_{2}, d$-TFA, DMSO- $d_{6}$, or $\mathrm{D}_{2} \mathrm{O}$ on a Bruker Fourier spectrometer equipped with a direct observe probehead operating at a proton frequency of $300 \mathrm{MHz}$. Sample temperature was set to $298 \mathrm{~K}$. Chemical shifts are given in parts per million (ppm, $[\delta])$ and were referenced by using the residual signal of the deuterated solvent.

\section{Solid state NMR}

${ }^{13} \mathrm{C}$ solid-state magic angle spinning (ssMAS) NMR spectra were either acquired utilizing cross polarization with a contact time of $2 \mathrm{~ms}$ and a spinning frequency of $15 \mathrm{kHz}$ or in the case of PAA- $b$-P $t$ BAA utilizing direct excitation and proton decoupling and a spinning frequency of $5 \mathrm{kHz}$. All data were collected on a Bruker Avance III HD $400 \mathrm{MHz}$ spectrometer equipped with a $4 \mathrm{~mm}$ dual channel probe. Sample temperature was set to $303 \mathrm{~K}$. The carbon chemical shifts were referenced externally, setting the high-frequency (methylene) signal of adamantane to $38.5 \mathrm{ppm}^{34}$

\section{Size-exclusion chromatography (SEC)}

SEC was performed on a Shimadzu system (Shimadzu Deutschland GmbH, Duisburg, Germany) equipped with a SCL-10A system controller, a LC-10AD pump, and a RID-10A refractive index detector using a solvent mixture containing chloroform $\left(\mathrm{CHCl}_{3}\right)$, triethylamine (TEA), and iso-propanol (i-PrOH) $(94: 4: 2)$ at a flow rate of $1 \mathrm{~mL} \mathrm{~min}^{-1}$ on a PSS SDV linear $\mathrm{S}(5 \mu \mathrm{m})$ column at $40{ }^{\circ} \mathrm{C}$ (Polymer Standards Service GmbH, PSS, Mainz, Germany). The system was calibrated with narrowly distributed PS (310-128 $000 \mathrm{~g} \mathrm{~mol}^{-1}$ ) standards (PSS, Mainz, Germany).

\section{Water based SEC}

SEC was performed on a Jasco system equipped with a DG-2080-53 degasser, PU-980 pump, and a RI-2031 Plus refractive index detector (Jasco Deutschland Labor- und Datentechnik GmbH, Groß-Umstadt, Germany) using $0.1 \mathrm{M}$ $\mathrm{Na}_{2} \mathrm{HPO}_{4} / 0.05 \% \mathrm{NaN}_{3} \mathrm{pH} 9$ as eluent at a flow rate of $1 \mathrm{~mL}$ $\min ^{-1}$ on a column set of PSS SUPREMA $1000 \AA$ and $30 \AA$ $(10 \mu \mathrm{m})$ at $30^{\circ} \mathrm{C}$ (PSS, Mainz, Germany).

\section{Zeta-potential measurements}

The samples for the zeta-potential measurements were prepared by titration of a $1 \mathrm{~g} \mathrm{~L}^{-1}$ solution of $\mathrm{PAA}_{25}-b$-PDha ${ }_{50}$ in 0.1 $\mathrm{N} \mathrm{HCl}$ with $0.1 \mathrm{~N} \mathrm{NaOH}$. For the titration and $\mathrm{pH}$ detection, a Metrohm 765 Dosimat titrator with a Greisinger electronic GMH3539 digital pH-/mV-electrode with thermometer was used. $1 \mathrm{~mL}$ samples for the measurements were taken at the desired $\mathrm{pH}$ values. The $\zeta$-potentials were measured using a Zetasizer Nano ZS from Malvern (Malvern Instruments $\mathrm{GmbH}$ Gültstein, Germany) via M3-PALS technique with a $\mathrm{He}-\mathrm{Ne}$ 
laser operating at $633 \mathrm{~nm}$. The detection angle was $13^{\circ}$. The electrophoretic mobilities $(u)$ were converted into $\zeta$-potentials via the Smoluchowski equation. ${ }^{35}$

$$
\zeta=\frac{u \eta}{\varepsilon}
$$

where $\eta$ denotes the viscosity and $\varepsilon$ the permittivity of the solution.

\section{Synthesis of tert-butoxycarbonylaminomethyl acrylate (tBAMA)}

$N$-(tert-Butoxycarbonyl)-D-serine methyl ester (10 g, $45.6 \mathrm{mmol})$ was dissolved in dichloromethane $(200 \mathrm{~mL})$. Methanesulfonyl chloride (Ms-Cl; $6 \mathrm{~mL}, 77.5 \mathrm{mmol}$ ) was added to the solution under vigorous stirring. The reaction mixture was cooled to $0{ }^{\circ} \mathrm{C}$ and triethylamine (TEA, $23 \mathrm{~mL}, 165.9 \mathrm{mmol}$ ) was added drop-wise. The solution was stirred at $0^{\circ} \mathrm{C}$ for $1 \mathrm{~h}$, and further $2 \mathrm{~h}$ at room temperature. Then the reaction mixture was washed with a potassium bisulfate solution (1\%) to neutrality. The organic phase was dried over $\mathrm{Na}_{2} \mathrm{SO}_{4}$, filtered and the solvent removed under reduced pressure. The product was further purified via column chromatography with silica gel (hexane/ethyl acetate v/v 8/2). The product was dried under reduced pressure obtaining a colorless oil in a yield of $87 \%$ (8 g, $39 \mathrm{mmol})$.

${ }^{1} \mathrm{H}-\mathrm{NMR}\left(300 \mathrm{MHz}, \mathrm{CDCl}_{3}, \delta\right): 7.00(\mathrm{~s}, 1 \mathrm{H},-\mathrm{NH}), 6.13(\mathrm{~s}$, $1 \mathrm{H},-\mathrm{C}=\mathrm{CH}-), 5.70(\mathrm{~s}, 1 \mathrm{H}-\mathrm{C}=\mathrm{CH}-), 3.8\left(\mathrm{~s}, 3 \mathrm{H},-\mathrm{O}-\mathrm{CH}_{3}-\right)$, 1.46 (s, $\left.9 \mathrm{H},-\mathrm{COO}-\mathrm{C}\left(\mathrm{CH}_{3}\right)_{3}\right) \mathrm{ppm} ;{ }^{13} \mathrm{C}-\mathrm{NMR}\left(75 \mathrm{MHz}_{\mathrm{CDCl}}, \delta\right)$ : $164.57\left(-\mathrm{COO}-\mathrm{CH}_{3}\right), 152.82\left(-\mathrm{COO}-\mathrm{C}\left(\mathrm{CH}_{3}\right)_{3}\right), 131.13\left(\mathrm{CH}_{2}=C\right)$, $105.28\left(\mathrm{CH}_{2}=\mathrm{C}\right), 80.58\left(-\mathrm{COO}-\mathrm{C}\left(\mathrm{CH}_{3}\right)_{3}\right), 52.98\left(-\mathrm{COO}-\mathrm{CH}_{3}\right)$, $28.45\left(-\mathrm{COO}-\mathrm{C}\left(\mathrm{CH}_{3}\right)_{3}\right) \mathrm{ppm}$.

The block copolymers $\mathrm{PS}_{30}-b$-P $t \mathrm{BAMA}_{40}$ and $\mathrm{P}_{n} \mathrm{BA}_{25}-b$ P BAMA $_{50}$ were synthesized according to a recently published procedure. $^{28}$

\section{Synthesis of $\mathbf{P S}_{30}-b-\left(\right.$ PAMA $_{10}-c o-$ PDha $\left._{30}\right)$}

$\mathrm{PS}_{30}-b-\mathrm{P}_{\mathrm{BAMA}} \mathrm{BA}_{40}(300 \mathrm{mg})$ was dissolved in dichloromethane (5 mL). Afterwards, trifluoroacetic acid $(1.15 \mathrm{~mL}, 0.0149 \mathrm{~mol}$, 10 eq. per monomer unit) was added and the reaction was stirred at RT for $19 \mathrm{~h}$. Then, the reaction mixture was precipitated in $40 \mathrm{~mL}$ hexane. The isolated block copolymer $(257 \mathrm{mg})$ was dried in vacuo.

${ }^{1} \mathrm{H}$-NMR (300 MHz, d-TFA, $\delta$ ): 8.00-6.20 (ArH), 4.45-3.85 $\left(-\mathrm{COOCH}_{3}\right.$ ), 3.60-2.40 (overlapping $-\mathrm{NH}_{3}{ }^{+},-\mathrm{CH}_{2}-\mathrm{CH}-,-\mathrm{CH}_{2}-$ $\left.\mathrm{CH}-,-\mathrm{CH}_{2}-\mathrm{C}-\right), 1.50\left(-\mathrm{NH}-\mathrm{COOC}\left(\mathrm{CH}_{3}\right)_{3}\right) ;{ }^{13} \mathrm{C}-\mathrm{NMR}(75 \mathrm{MHz}, d-$ TFA, $\delta$ ): 173.87 (-COO- $\left.\mathrm{CH}_{3}\right), 156.16(-A r-C$, overlapping with solvent signals), $59.12\left(-\mathrm{CH}_{2}-\mathrm{CH}-,-\mathrm{CH}_{2}-\mathrm{C}-\right)$, 50.00 (-COO$\left.\mathrm{CH}_{3}\right), 39.13\left(-\mathrm{CH}_{2}-\mathrm{CH}-,-\mathrm{CH}_{2}-\mathrm{C}^{-}\right) \mathrm{ppm}$.

\section{Synthesis of $\mathrm{PS}_{30}-b-\left(\mathrm{P} t \mathrm{BAMA}_{3}-\mathrm{co}-\mathrm{PDha}_{37}\right)$}

$\mathrm{P}_{30}-b$-P $t$ BAMA 40 (363 mg) was dissolved in dimethyl sulfoxide $(6 \mathrm{~mL})$ and water $(0.5 \mathrm{~mL})$. Afterwards, $\mathrm{LiOH} \cdot \mathrm{H}_{2} \mathrm{O}(1.65 \mathrm{~g}$, 21 eq. per monomer unit) was suspended in the solution and the reaction mixture was heated to $100{ }^{\circ} \mathrm{C}$ for $22 \mathrm{~h}$. After cooling down to RT and centrifugation, the precipitated block copolymer was isolated and mixed with water $(10 \mathrm{~mL})$, before the solution was neutralized with $\mathrm{HCl}_{\mathrm{aq}}$. For further purifi- cation, the solution was dialyzed against water. Afterwards, the block copolymer was dried in vacuo $(210 \mathrm{mg})$.

${ }^{1} \mathrm{H}$-NMR (300 MHz, d-TFA, $\delta$ ): 7.30-6.30 (ArH), 2.40-1.30 $\left(-\mathrm{CH}_{2}-\mathrm{CH}-,-\mathrm{CH}_{2}-\mathrm{CH}-,-\mathrm{CH}_{2}-\mathrm{C}-\right), 2.20-1.18\left(-\mathrm{COO}\left(\mathrm{CH}_{3}\right)_{3}\right)$; ${ }^{13} \mathrm{C}-\mathrm{NMR}$ (75 MHz, solid state, $\delta$ ): $179.34\left(-\mathrm{COOH},-\mathrm{COOCH}_{3}\right)$, $157.26\left(-\mathrm{NH}-\mathrm{COO}-\left(\mathrm{CH}_{3}\right)_{3}\right), \quad 146.37 / 128.53$ (-Ar-C-), 61.99 $\left(-\mathrm{CH}_{2}-\mathrm{C}-,-\mathrm{CH}_{2}-\mathrm{CH}-\right), 46.27$ (- $\left.\mathrm{CH}_{2}-\mathrm{C}-\right), 42.34\left(-\mathrm{CH}_{2}-\mathrm{CH}-\right)$, $30.24\left(-\mathrm{COO}\left(\mathrm{CH}_{3}\right)_{3}\right)$ ppm.

\section{Synthesis of $\mathrm{PS}_{30}-b$-PDha 40}

$\mathrm{PS}_{30}-b$-(PtBAMA 3 - $\left.\mathrm{Co}-\mathrm{PDha}_{37}\right)$ (100 mg) was dissolved in water $(10 \mathrm{~mL})$ and trifluoroacetic acid $(1 \mathrm{~mL}, 0.0129 \mathrm{~mol}, 26 \mathrm{eq}$. per monomer unit) was added. The reaction mixture was stirred for $96 \mathrm{~h}$ and subsequently neutralized using sodium hydroxide solution (0.2 M). Afterwards, the dissolved block copolymer was further purified by dialysis against water. The isolated block copolymer was dried in vacuo $(107 \mathrm{mg})$.

${ }^{1} \mathrm{H}-\mathrm{NMR}$ (300 MHz, d-TFA, $\delta$ ): 7.30-6.30 (ArH), 1.30-2.40 $\left(-\mathrm{CH}_{2}-\mathrm{CH}-,-\mathrm{CH}_{2}-\mathrm{CH}-,-\mathrm{CH}_{2}-\mathrm{C}-\right)$; ${ }^{13} \mathrm{C}-\mathrm{NMR}$ (75 MHz, solid state, $\delta): 179.34\left(-\mathrm{COOH},-\mathrm{COOCH}_{3}\right), 146.37 / 128.53(-\mathrm{Ar}-\mathrm{C}-)$, $61.99\left(-\mathrm{CH}_{2}-\mathrm{C}-,-\mathrm{CH}_{2}-\mathrm{CH}-\right), 46.27\left(-\mathrm{CH}_{2}-\mathrm{C}-\right), 42.34\left(-\mathrm{CH}_{2}-\right.$ CH-) ppm.

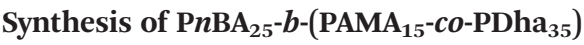

$\mathrm{P}_{n} \mathrm{BA}_{25}-b$-P $t$ BAMA $_{50}$ (300 mg) was dissolved in dichloromethane $(5 \mathrm{~mL})$. Afterwards, trifluoroacetic acid $(1.15 \mathrm{~mL}$, $0.0149 \mathrm{~mol}, 10 \mathrm{eq}$. per monomer unit) was added and the reaction was stirred at $50{ }^{\circ} \mathrm{C}$ for $1 \mathrm{~h}$. After cooling down to RT, the reaction mixture was precipitated in $40 \mathrm{~mL}$ hexane. The isolated block copolymer (176 mg) was dried in vacuo.

${ }^{1} \mathrm{H}-\mathrm{NMR} \quad\left(300 \mathrm{MHz}, \mathrm{CD}_{2} \mathrm{Cl}_{2}, \delta\right): 4.15\left(-\mathrm{COOCH}_{2}-\right), 2.39$ (- $\left.\mathrm{CH}_{2}-\mathrm{CH}-\right), 1.98\left(-\mathrm{CH}_{2}-\mathrm{CH}-\right), 1.65$ (-COO- $\left.\mathrm{CH}_{2}-\mathrm{CH}_{2}-\right), 1.45$ $\left(-\mathrm{CH}_{2}-\mathrm{CH}_{3}\right), 0.95\left(-\mathrm{CH}_{2}-\mathrm{CH}_{3}\right) ;{ }^{13} \mathrm{C}-\mathrm{NMR}\left(75 \mathrm{MHz}, \mathrm{CD}_{2} \mathrm{Cl}_{2}, \delta\right)$ : $176.52\left(-\mathrm{COOCH}_{2}-\right), 66.15\left(-\mathrm{COOCH}_{2}-\right), 43.46\left(-\mathrm{CH}_{2}-\mathrm{CH}-\right)$, $37.00\left(-\mathrm{CH}_{2}-\mathrm{CH}-\right), 32.33\left(-\mathrm{CH}_{2}-\mathrm{CH}_{2}-\mathrm{CH}_{2}-\mathrm{CH}_{3}\right), 21.09\left(-\mathrm{CH}_{2}-\right.$ $\left.\mathrm{CH}_{2}-\mathrm{CH}_{2}-\mathrm{CH}_{3}\right), 15.37\left(-\mathrm{CH}_{2}-\mathrm{CH}_{2}-\mathrm{CH}_{2}-\mathrm{CH}_{3}\right)$ ppm.

Under these conditions, only signals corresponding to the PnBA segment of the block copolymer can be detected. Hence, we also conducted NMR studies in deuterated TFA:

${ }^{1} \mathrm{H}-\mathrm{NMR} \quad(300 \mathrm{MHz}, \quad d$-TFA, $\delta): 4.15 \quad\left(-\mathrm{COOCH}_{2}-\right), 3.95$ (-COOCH$H_{3}$ ), 3.4-2.4 (overlaying $\mathrm{NH}_{3}{ }^{+}$and PAMA-backbone), 2.39 ( $\left.-\mathrm{CH}_{2}-\mathrm{CH}-\right), 1.98\left(-\mathrm{CH}_{2}-\mathrm{CH}-\right), 1.85$ (-COO- $\left.\mathrm{CH}_{2}-\mathrm{CH}_{2}-\right)$, $1.45\left(-\mathrm{CH}_{2}-\mathrm{CH}_{3}\right), 1.10\left(-\mathrm{CH}_{2}-\mathrm{CH}_{3}\right) ;{ }^{13} \mathrm{C}-\mathrm{NMR}$ (75 MHz, d-TFA, $\delta): 180.52\left(-\mathrm{COOCH}_{2}-\right), 180.34(-\mathrm{COO}-), 66.15\left(-\mathrm{COOCH}_{2}-\right.$, $\left.-\mathrm{CH}_{2}-\mathrm{C}-,-\mathrm{CH}_{2}-\mathrm{CH}-\right), 65.00\left(-\mathrm{COOCH}_{3}\right), 43.46\left(-\mathrm{CH}_{2}-\mathrm{CH}-\right)$, $37.00\left(-\mathrm{CH}_{2}-\mathrm{C}\right), 32.33\left(-\mathrm{CH}_{2}-\mathrm{CH}_{2}-\mathrm{CH}_{2}-\mathrm{CH}_{3}\right), 21.09\left(-\mathrm{CH}_{2}-\right.$ $\left.\mathrm{CH}_{2}-\mathrm{CH}_{2}-\mathrm{CH}_{3}\right), 15.37\left(-\mathrm{CH}_{2}-\mathrm{CH}_{2}-\mathrm{CH}_{2}-\mathrm{CH}_{3}\right) \mathrm{ppm}$.

\section{Synthesis of $\mathrm{PnBA}_{25}-b-\left(\mathrm{PtBAA}_{20}-c o-\mathrm{PDha}_{30}\right)$}

$\mathrm{P} n \mathrm{BA}_{40}-b$-PtBAMA ${ }_{50}(300 \mathrm{mg})$ was dissolved in DMSO (5 mL). Afterwards, $\mathrm{LiOH} \cdot \mathrm{H}_{2} \mathrm{O}(1.32 \mathrm{~g}, 21 \mathrm{eq}$. per monomer unit) was suspended in the solution and the reaction mixture was heated to $80{ }^{\circ} \mathrm{C}$ for $3 \mathrm{~h}$. After cooling down to RT the precipitated block copolymer was mixed with water $(10 \mathrm{~mL})$, and the dispersion was neutralized with $\mathrm{HCl}_{\mathrm{aq}}$. After dialysis against water, the block copolymer was dried in vacuo (159 mg). 
${ }^{1} \mathrm{H}-\mathrm{NMR}\left(300 \mathrm{MHz}, \mathrm{D}_{2} \mathrm{O}, \delta\right): 4.0$ (-COO- $\mathrm{CH}_{2}-$ ), 2.40 (overlapping, - $\left.\left.\mathrm{CH}_{2}-\mathrm{CH}-, \quad-\mathrm{CH}_{2}-\mathrm{C}-,-\mathrm{CH}_{2}-\mathrm{CH}-\right)\right), 1.40 \quad(-\mathrm{NH}-\mathrm{COO}$ $\left(\mathrm{CH}_{3}\right)_{3}, \quad-\mathrm{COO}-\mathrm{CH}_{2}-\mathrm{CH}_{2}-\mathrm{CH}_{2}-\mathrm{CH}_{3}, \quad-\mathrm{COO}-\mathrm{CH}_{2}-\mathrm{CH}_{2}-\mathrm{CH}_{2}-$ $\left.\mathrm{CH}_{3}\right), 0.95\left(-\mathrm{CH}_{2}-\mathrm{CH}_{3}\right) ;{ }^{13} \mathrm{C}-\mathrm{NMR}\left(75 \mathrm{MHz}, \mathrm{D}_{2} \mathrm{O}, \delta\right): 176.52$ (-CH-COO-), 174.14 (-C-COOH), $153.59\left(-\mathrm{NH}-\mathrm{COO}\left(\mathrm{CH}_{3}\right)_{3}\right)$, $81.20\left(-\mathrm{C}\left(\mathrm{CH}_{3}\right)\right), 66.10\left(-\mathrm{COO}-\mathrm{CH}_{2}, \mathrm{CH}_{2}-\mathrm{CH}-\right), 60.21\left(-\mathrm{CH}_{2}-\right.$ $\mathrm{C}-), 41.2\left(-\mathrm{CH}_{2}-\mathrm{C}-\right), 38.00\left(-\mathrm{CH}_{2}-\mathrm{CH}-\right), 32\left(-\mathrm{COO}-\mathrm{CH}_{2}-\mathrm{CH}_{2}-\right)$, $28.00\left(-\mathrm{NH}-\mathrm{COO}\left(\mathrm{CH}_{3}\right)_{3}\right), 19.00\left(-\mathrm{CH}_{2}-\mathrm{CH}_{3}\right), 12.00\left(-\mathrm{CH}_{2}-\mathrm{CH}_{3}\right)$ ppm.

Under these conditions, mainly signals corresponding to the PnBA segment of the block copolymer can be detected. Hence, we also conducted NMR studies in deuterated TFA:

${ }^{1} \mathrm{H}$-NMR (300 MHz, d-TFA, $\delta$ ): 4.50 (-COO-CH $2_{2}^{-}$), 3.60-2.60 (overlapping $\left.\mathrm{NH}_{3}{ }^{+},-\mathrm{CH}_{2}-\mathrm{C}-\right), 2.45\left(-\mathrm{CH}_{2}-\mathrm{CH}-\right), 2.20\left(-\mathrm{CH}_{2}-\right.$ $\mathrm{CH}-), 1.98$ (-COO- $\left.\mathrm{CH}_{2}-\mathrm{CH}_{2}-\right), 1.85\left(-\mathrm{CH}_{2}-\mathrm{CH}_{3}\right), 1.65(-\mathrm{NH}-$ $\left.\mathrm{COO}\left(\mathrm{CH}_{3}\right)_{3}\right), 1.2\left(-\mathrm{CH}_{2}-\mathrm{CH}_{3}\right) ;{ }^{13} \mathrm{C}-\mathrm{NMR}$ (75 MHz, d-TFA, $\left.\delta\right):$ 179.24 (-CH-COO-), 179.14 (-C-COOH), 161.00 (overlapping with solvent signal, $\left.-\mathrm{NH}-\mathrm{COO}\left(\mathrm{CH}_{3}\right)_{3}\right), 92.00\left(-C\left(\mathrm{CH}_{3}\right)\right), 70.10$ (-COO- $\left.\mathrm{CH}_{2}\right), 65.21\left(-\mathrm{CH}_{2}-\mathrm{C}-,-\mathrm{CH}_{2}-\mathrm{CH}-\right), 42.00\left(-\mathrm{CH}_{2}-\mathrm{CH}-\right)$, $40.00\left(-\mathrm{CH}_{2}-\mathrm{C}-\right), 37.00\left(-\mathrm{NH}-\mathrm{COO}\left(\mathrm{CH}_{3}\right)_{3}\right), 28.7\left(-\mathrm{CH}_{2}-\mathrm{CH}_{2}-\right.$ $\left.\mathrm{CH}_{3}\right), 19\left(-\mathrm{CH}_{2}-\mathrm{CH}_{3}\right), 15.00\left(-\mathrm{CH}_{2}-\mathrm{CH}_{3}\right) \mathrm{ppm}$.

\section{Synthesis of $\mathrm{PAA}_{25}-\boldsymbol{b}$-PDha 5}

$\mathrm{P}_{n} \mathrm{BA}_{25}-b$-(PtBAA $\left.20-c o-\mathrm{PDha}_{30}\right)(100 \mathrm{mg})$ was dissolved in water $(10 \mathrm{~mL})$ and afterwards trifluoroacetic acid $(1 \mathrm{~mL}, 0.0129 \mathrm{~mol}$, 26 eq. per monomer unit) was added. The reaction mixture was stirred for $96 \mathrm{~h}$ at RT, and then neutralized using a sodium hydroxide solution $(0.2 \mathrm{M})$. Afterwards, the dissolved block copolymer was further purified by dialysis against water and dried in vacuo (112 $\mathrm{mg})$.

${ }^{1} \mathrm{H}-\mathrm{NMR}\left(300 \mathrm{MHz}, \mathrm{D}_{2} \mathrm{O}, \delta\right): 3.2-1.2\left(-\mathrm{CH}_{2}-\mathrm{CH}-,-\mathrm{CH}_{2}-\mathrm{CH}-\right.$, $\left.-\mathrm{CH}_{2}-\mathrm{C}-\right),{ }^{13} \mathrm{C}-\mathrm{NMR}$ (75 $\left.\mathrm{MHz}, \mathrm{D}_{2} \mathrm{O}, \delta\right): 184.01$ (-C-COOH), $179.42(-\mathrm{CH}-\mathrm{COOH}), 61.50\left(-\mathrm{CH}_{2}-\mathrm{C}-\right), 59.40\left(-\mathrm{CH}_{2}-\mathrm{CH}-\right)$, $45.06\left(-\mathrm{CH}_{2}-\mathrm{C}-\right), 43.64\left(-\mathrm{CH}_{2}-\mathrm{CH}-\right)$ ppm.

\section{Results and discussion}

We herein report the synthesis and solution behavior of block copolymers containing a polydehydroalanine (PDha) segment and (at that stage) either a hydrophobic PS or a hydrophilic PAA block. We are interested in such materials as, depending on the co-block, either amphiphilic or double hydrophilic block copolymers with pH-dependent charge characteristics are obtained and these could serve as interesting building blocks in interpolyelectrolyte complexes (IPECs). The materials are synthesized using sequential atom transfer radical polymerization (ATRP) of styrene/ $n$-butyl acrylate, followed by $t$ BAMA, as reported recently. ${ }^{28}$ Subsequently, deprotection of either the carboxyl moiety or the amino functionality is carried out, followed by conversion to polyampholytic PDha-containing block copolymers (Schemes 1 and 2). First, macroinitiators of $\mathrm{PS}_{30}-\mathrm{Br}$ and $\mathrm{P}_{n} \mathrm{BA}_{25}-\mathrm{Br}$ were synthesized (the subscripts denote the corresponding degree of polymerization).

Subsequent block extension in anisole at $50{ }^{\circ} \mathrm{C}$ yielded $\mathrm{PS}_{30}-b$-P $t$ BAMA $_{40}$ and $\mathrm{P} \mathrm{BA}_{25}-b$-PtBAMA 50 block copolymers with narrow dispersity (Table 1 ). The composition was deter-

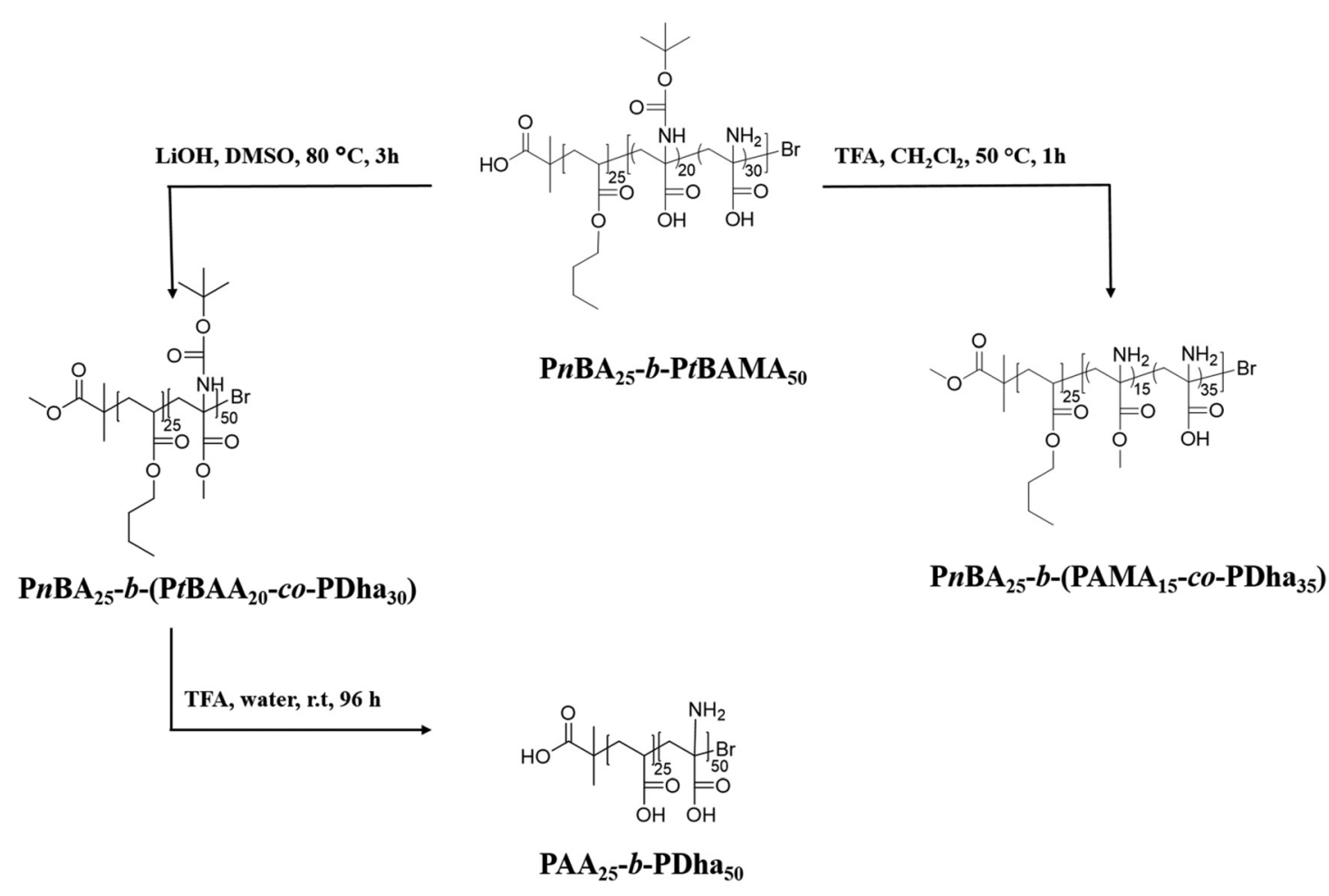

Scheme 1 Structure of $\mathrm{PnBA}_{25}-b$-PtBAMA 50 and the resulting block copolymers after the respective deprotection steps. 
LiOH, 1,4-dioxane/water, $100^{\circ} \mathrm{C}, 22 \mathrm{hrs}$.

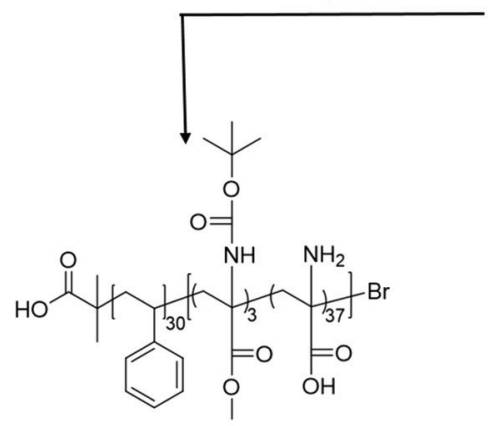

$\mathrm{PS}_{30}-b-\left(\mathrm{PtBAMA}_{3}-c o-\mathrm{PDha}_{37}\right)$

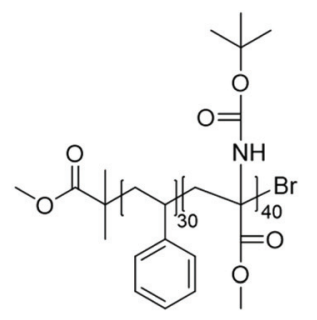

$\mathrm{PS}_{30}-b-\mathrm{P} t \mathrm{BAMA}$
TFA, $\mathrm{CH}_{2} \mathrm{Cl}_{2}$, r.t., 19 hrs.
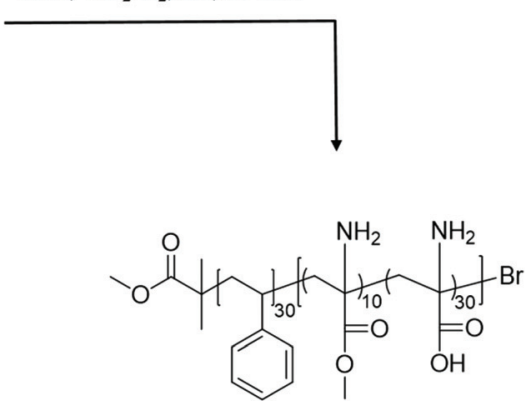

TFA, $\mathrm{CH}_{2} \mathrm{Cl}_{2}$, r.t., 96 hrs.

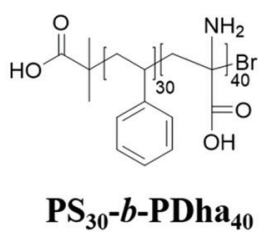

Scheme 2 Structure of $\mathrm{PS}_{30}-b$-PtBAMA 40 and the corresponding block copolymers after different deprotection steps.

Table 1 Characteristics of $\mathrm{PS}_{30}-b-\mathrm{PtBAMA}_{40}$ and $\mathrm{PnBA}_{25}-b-\mathrm{PtBAMA} \mathrm{A}_{50}$ and the resulting block copolymers after different deprotection steps (the subscripts denote the degree of polymerization of the corresponding block)

\begin{tabular}{|c|c|c|c|c|}
\hline Entry & (Block co-) polymer & $\begin{array}{l}M_{\mathrm{n}} \\
{\left[\mathrm{g} \mathrm{mol}^{-1}\right]}\end{array}$ & $\begin{array}{l}M_{\mathrm{n}, \text { theo }} \\
{\left[\mathrm{g} \mathrm{mol}^{-1}\right]}\end{array}$ & $Ð$ \\
\hline 1 & $\mathrm{P} n \mathrm{BA}_{25}-\mathrm{Br}$ & $3600^{a}$ & $3200^{a}$ & $1.13^{a}$ \\
\hline 2 & $\mathrm{P} \mathrm{BA}_{25}-b-\mathrm{P} t \mathrm{BAMA}_{50}$ & $8100^{a}$ & $23600^{a}$ & $1.18^{a}$ \\
\hline 3 & $\mathrm{P}_{n} \mathrm{BA}_{25}-b-\left(\mathrm{PAMA}_{15}-c o-\mathrm{PDha}_{35}\right)$ & - & 18500 & - \\
\hline 4 & $\mathrm{P} n \mathrm{BA}_{25}-b-\left(\mathrm{P} \mathrm{BAA}_{20}-c o-\mathrm{PDha}_{30}\right)$ & - & 20000 & - \\
\hline 5 & $\mathrm{PAA}_{25}-b-\mathrm{PDha}_{50}$ & $21800^{b}$ & $14900^{b}$ & $1.35^{b}$ \\
\hline 6 & $\mathrm{PS}_{30}-\mathrm{Br}$ & $3400^{a}$ & $3100^{a}$ & $1.10^{a}$ \\
\hline 7 & $\mathrm{PS}_{30}-b-\mathrm{P} t \mathrm{BAMA}_{40}$ & $7900^{a}$ & $23400^{a}$ & $1.38^{a}$ \\
\hline 8 & $\mathrm{PS}_{30}-b-\left(\mathrm{PAMA}_{10}-c o-\mathrm{PDha}_{30}\right)$ & - & 19400 & - \\
\hline 9 & $\mathrm{PS}_{30}-b-\left(\mathrm{P} t \mathrm{BAMA}_{3}-c o-\mathrm{PDha}_{37}\right)$ & - & 22800 & - \\
\hline 10 & $\mathrm{PS}_{30}-b-\mathrm{PDha}_{40}$ & - & 18800 & - \\
\hline
\end{tabular}

${ }^{a}$ Determined by SEC $\left(\mathrm{CHCl}_{3} / \mathrm{TEA} / \mathrm{i}-\mathrm{PrOH}:\right.$ 94/4/2). PS-calibration. ${ }^{b}$ Determined by SEC $0.1 \mathrm{M} \mathrm{Na}_{2} \mathrm{HPO}_{4} \mathrm{pH}$ 9. Pullulan calibration.

mined using ${ }^{1} \mathrm{H}$-NMR spectroscopy. Thereby, hydrolysis of $\mathrm{PS}_{30}-b$-P $t$ BAMA $_{40}$ will lead to amphiphilic block copolymers, whereas in the case of $\mathrm{P} \mathrm{BA}_{25}-b$-PtBAMA ${ }_{50}$ double hydrophilic $\mathrm{PAA}_{25}-b-\mathrm{PDha}_{40}$ will be obtained (Scheme 1).

\section{Synthesis of $\mathrm{PAA}_{25}-\boldsymbol{b}-\mathrm{PDha}_{50}$}

We have earlier reported on the (selective) deprotection of either the Boc protective group using TFA at $50{ }^{\circ} \mathrm{C}$ or the hydrolysis of the methyl ester of PtBAMA homopolymers at $100{ }^{\circ} \mathrm{C}$ using $\mathrm{LiOH} \cdot \mathrm{H}_{2} \mathrm{O} \cdot{ }^{29,30}$ However, during the course of these studies we observed that both for $\mathrm{P}_{n} \mathrm{BA}_{25}-b$-PtBAMA and $\mathrm{PS}_{30}-b$-PtBAMA 40 also significant deprotection of the other moiety occurred, e.g. treatment with TFA at RT removed both Boc group and the methyl ester. We therefore varied the conditions $\left(50{ }^{\circ} \mathrm{C}\right.$ and $1 \mathrm{~h}$ reaction time) under which these reactions have been carried out, although without obtaining a truly orthogonal procedure so far. Nevertheless, in our opinion the final amphiphilic and double hydrophilic block copolymers featuring PDha segments represent the most interesting materials and for selective deprotection of either $-\mathrm{COOH}$ or $-\mathrm{NH}_{2}$ presumably another combination of protective groups might be favorable, at least in the case of block copolymers. Below we describe the so far most selective reaction conditions whereas more detailed descriptions including ${ }^{1} \mathrm{H}-\mathrm{NMR}$ and ${ }^{13} \mathrm{C}-\mathrm{NMR}$ spectroscopy are discussed in the ESI (Fig. S1 and $\mathrm{S} 2 \dagger$ ).

We first start by partial hydrolysis of $\mathrm{P}_{n} \mathrm{BA}_{25}-b-\mathrm{P}_{2} \mathrm{BAMA}_{50}$. Starting from $\mathrm{P}_{n} \mathrm{BA}_{25}-b$-P $t \mathrm{BAMA}_{50}$, the Boc protective group was cleaved by adding TFA (10 eq. per monomer unit) in dichloromethane at $50{ }^{\circ} \mathrm{C}$ for $1 \mathrm{~h}^{36}$ After precipitation in hexane, the block copolymer was characterized by NMR (Fig. 1). As can be seen, the signal of the Boc group at $1.5 \mathrm{ppm}$ disappears $(\sim 100 \%)$, indicating complete cleavage. A new broad signal ranging from 2.4 to $3.4 \mathrm{ppm}$ can be found, indicating the presence of a protonated amine functionality. Further, the alkyl chain of the $n \mathrm{BA}$ ester $(4.15,1.85,1.45$ and $1.1 \mathrm{ppm}$ ) and the corresponding backbone signals (2.39 and $1.98 \mathrm{ppm}$ ) have not been affected, whereas the signal for the methyl ester of the former PtBAMA segment (3.95 ppm) decreased to approximately $30 \%$ intensity (resulting in $\mathrm{P} \mathrm{BA}_{25}$ $b$-( PAMA $_{15}$-co-PDha 35$)$ ). Obviously, the chosen conditions are 


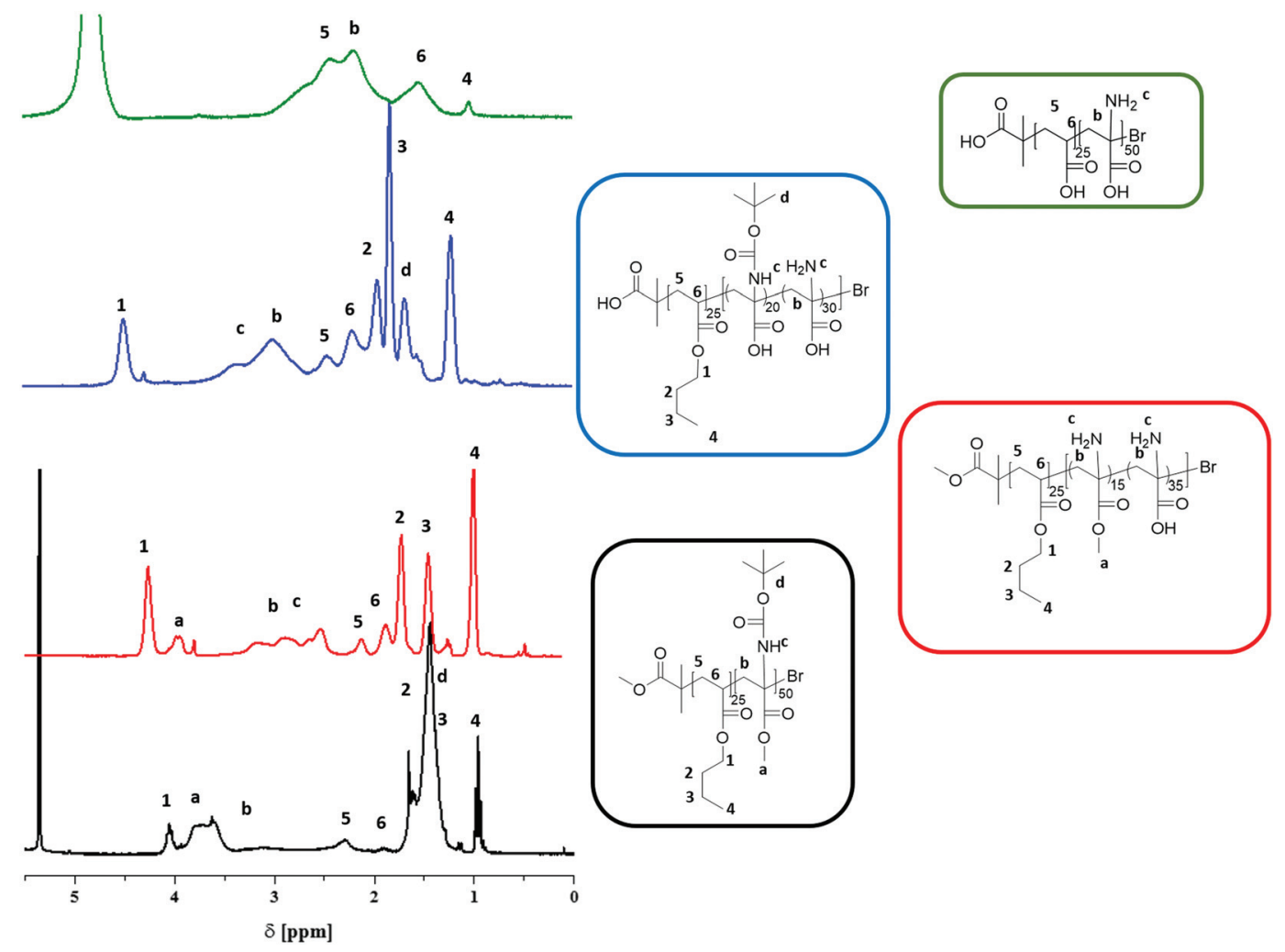

Fig. $1{ }^{1} \mathrm{H}-\mathrm{NMR}$ of $\mathrm{PnBA}_{25}-b-\mathrm{PtBAMA}_{50}$ (black line, $\mathrm{CDCl}_{3}$ ), $\mathrm{PnBA}_{25}-b-\left(\mathrm{PAMA}_{15}-\mathrm{co}-\mathrm{PDha}_{35}\right.$ ) (red line, d-TFA), PnBA $25-b-\left(\mathrm{PtBAA}_{20}-\mathrm{co}-\mathrm{PDh} \mathrm{P}_{30}\right)(\mathrm{blue}$ line, $D_{2} O$ ), $P A A_{25}-b$-PDha 50 (green line, $D_{2} O$ ), numbers indicate signals which have been allocated to PnBA/PAA, letters indicate signals related to PtBAMA, PAMA, PtBAA and PDha.

too harsh for the methyl ester, leading to partial cleavage. Compared to earlier studies, here the second block can be used as internal reference, thereby improving the accuracy of NMR characterization. The cleavage of the methyl ester (and the butyl ester of $\mathrm{P} n \mathrm{BA}$ ) was carried out at $80{ }^{\circ} \mathrm{C}$ for $3 \mathrm{~h}$ and using 21 eq. $\mathrm{LiOH} \cdot \mathrm{H}_{2} \mathrm{O} \cdot{ }^{29,37}$ After neutralization using $\mathrm{HCl}_{\mathrm{aq}}$ and subsequent dialysis against water, ${ }^{1} \mathrm{H}$-NMR in deuterated TFA showed typical signals for $\mathrm{P} n \mathrm{BA}(4.5,2.45,2.20,1.98$, 1.85 and $1.2 \mathrm{ppm}$ ) and PtBAA (3 ppm and $1.65 \mathrm{ppm})$. The intensity of the Boc group at $1.5 \mathrm{ppm}$ decreased to $30 \%$, again

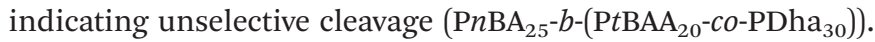
The removal of the Boc protective group during treatment with $\mathrm{LiOH} \cdot \mathrm{H}_{2} \mathrm{O}$ was already observed. In further studies, simultaneous cleavage of the Boc and methyl ester in $N$-Boc alanine methyl ester was justified by the possibility of water to act as dual acid/base catalyst. ${ }^{38,39}$

Subsequent full deprotection was achieved by treatment of $\mathrm{P}_{n} \mathrm{BA}_{25}-b$-( $\left.\mathrm{P}^{-} \mathrm{BAA}_{20}-c o-\mathrm{PDha}_{30}\right)$ with TFA at RT in water for $96 \mathrm{~h}$. The resulting $\mathrm{PAA}_{25}-b$-PDha 50 block copolymer is well soluble in water and ${ }^{1} \mathrm{H}-\mathrm{NMR}$ in $\mathrm{D}_{2} \mathrm{O}$ shows only signals for the polymer backbone (3.2-1.2 ppm), with the exception that a small fraction of remaining P $n$ BA can still be observed (below 10\%).

The results obtained by ${ }^{1} \mathrm{H}-\mathrm{NMR}$ were confirmed by ${ }^{13} \mathrm{C}$-NMR (Fig. 2). For P $n$ BA- $b$-PtBAMA, the signals of the backbone (61 and $41 \mathrm{ppm}$ ), the carbonyl group of PnBA (172 ppm), the methyl ester of PtBAMA (172 ppm) and the Boc protective group (153 ppm, 79 ppm, $28 \mathrm{ppm}$ ) can be observed. The signals of the butyl group of $\mathrm{P} n \mathrm{BA}$ can be detected at 64, 30, 19 and $13 \mathrm{ppm}$ and the methyl ester at $52 \mathrm{ppm}$. For $\mathrm{P}_{n \mathrm{BA}_{25}-b}-$ $\left(\mathrm{PAMA}_{15}-\mathrm{Co}-\mathrm{PDha}_{35}\right.$, measured in $d$-TFA) the Boc group (153, $79,28 \mathrm{ppm}$ ) is absent and the methyl ester (65 ppm) can still \left. be detected. For ${\mathrm{P} n \mathrm{BA}_{25}-b-\left(\mathrm{P} t \mathrm{BAA}_{20}-c o-\mathrm{PDha}\right.}_{30}\right)$, the signals for the carbonyl moieties could be detected (179.24 ppm and $179.14 \mathrm{ppm}$ ) and even the methyl groups of the Boc group (37 ppm) are visible. Also, both $\mathrm{P} n \mathrm{BA}(70,65,42,28,19$ and $15 \mathrm{ppm})$ and PtBAA (161, 92, 70 and $42 \mathrm{ppm})$ can be clearly identified. For PAA- $b$-PDha, mainly carbon atoms of the backbone (61, 59, 45 and $43 \mathrm{ppm}$ ) as well as the carbonyl groups (184 and $179 \mathrm{ppm}$ ) of the acid functions are observed (NMR in $\mathrm{D}_{2} \mathrm{O}$ ). The end group of P $n$ BA- $b$-PtBAMA (and also for PS- $b$ P $t$ BAMA) could not be detected in NMR spectroscopy. However, almost complete re-initiation of PtBAMA prepared via ATRP was possible, hinting to a good endgroup fidelity. ${ }^{28}$ During the deprotection steps, we expect to hydrolyze both the terminal bromine as well as the ester moiety of the ATRP initiator.

Differences in solubility of the purified intermediate stages are discussed in the ESI. $\dagger$ It was possible to measure NMR of $\mathrm{P}_{n} \mathrm{BA}_{25}-b$-( $\left.\mathrm{PAMA}_{15}-c o-\mathrm{PDha}_{35}\right)$ in DCM and TFA as well as $\mathrm{P}_{n} \mathrm{BA}_{25}-b-\left(\mathrm{P}^{2} \mathrm{BAA}_{20}-c o-\mathrm{PDha}_{30}\right)$ in TFA and in $\mathrm{D}_{2} \mathrm{O}$ (Fig. S3-S6 $\dagger$ ). After complete deprotection, SEC $\left(0.1 \mathrm{M} \mathrm{Na}_{2} \mathrm{HPO}_{4} / \mathrm{NaN}_{3} \mathrm{pH} 9\right.$, pullulan calibration) of $\mathrm{PAA}_{25}-b$-PDha ${ }_{50}$ showed a monomodal 

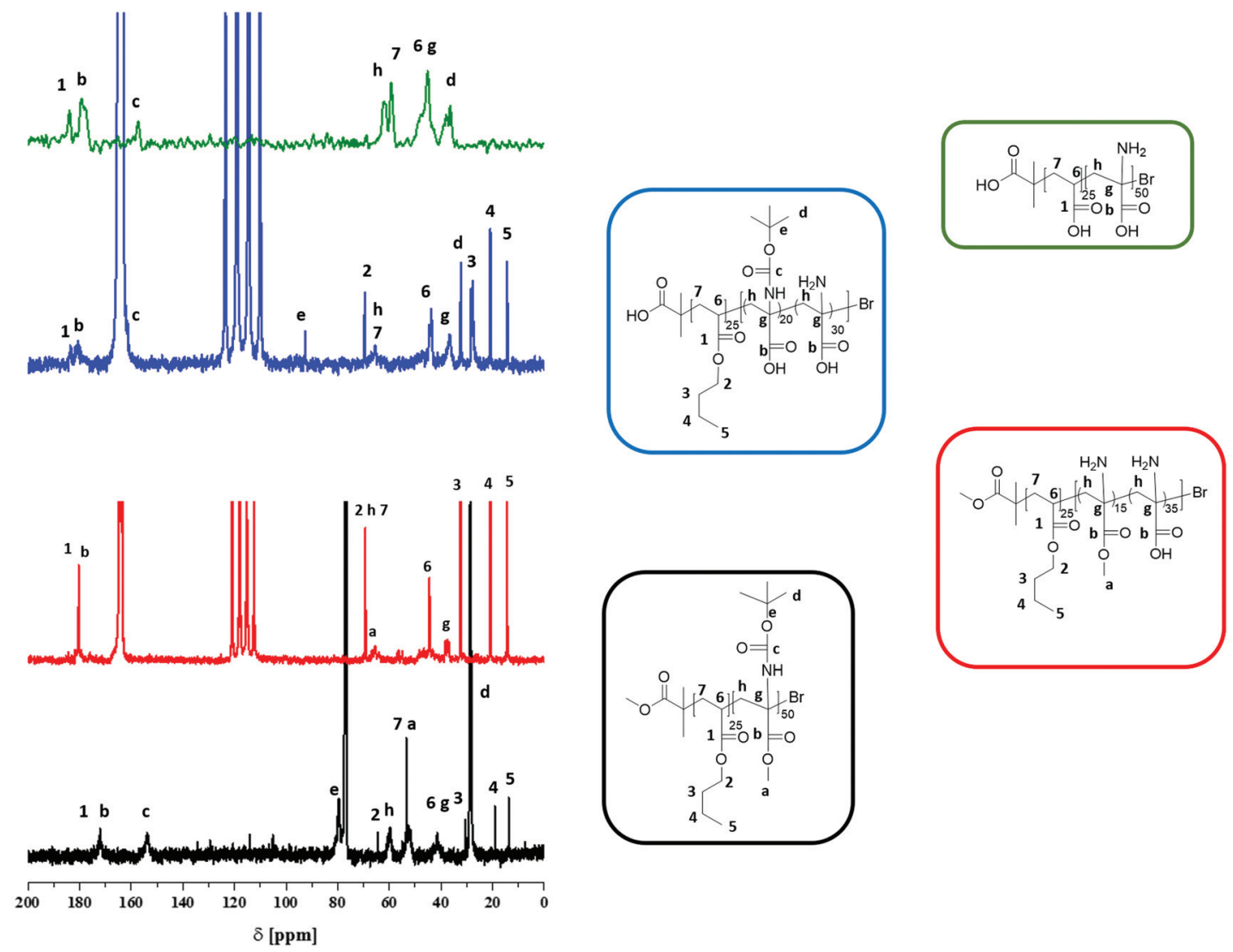

Fig. $2{ }^{13} \mathrm{C}-\mathrm{NMR}$ of $\mathrm{PnBA}_{25}-b$-PtBAMA 50 (black line, $\mathrm{CDCl}_{3}$ ), $\mathrm{PnBA}_{25}-b-\left(\mathrm{PAMA}_{15}-\mathrm{Co}-\mathrm{PDha}_{35}\right.$ ) (red line, d-TFA), PnBA $25-b-(\mathrm{PtBAA} 20-\mathrm{Co}-\mathrm{PDha} 30$ ) (blue line, $D_{2} O$ ), $P_{2 A}-b-P D h a_{50}$ (green line, $D_{2} O$ ), numbers indicate signals of PnBA/PAA, letters indicate signals of PtBAMA, PAMA, PtBAA and PDha.
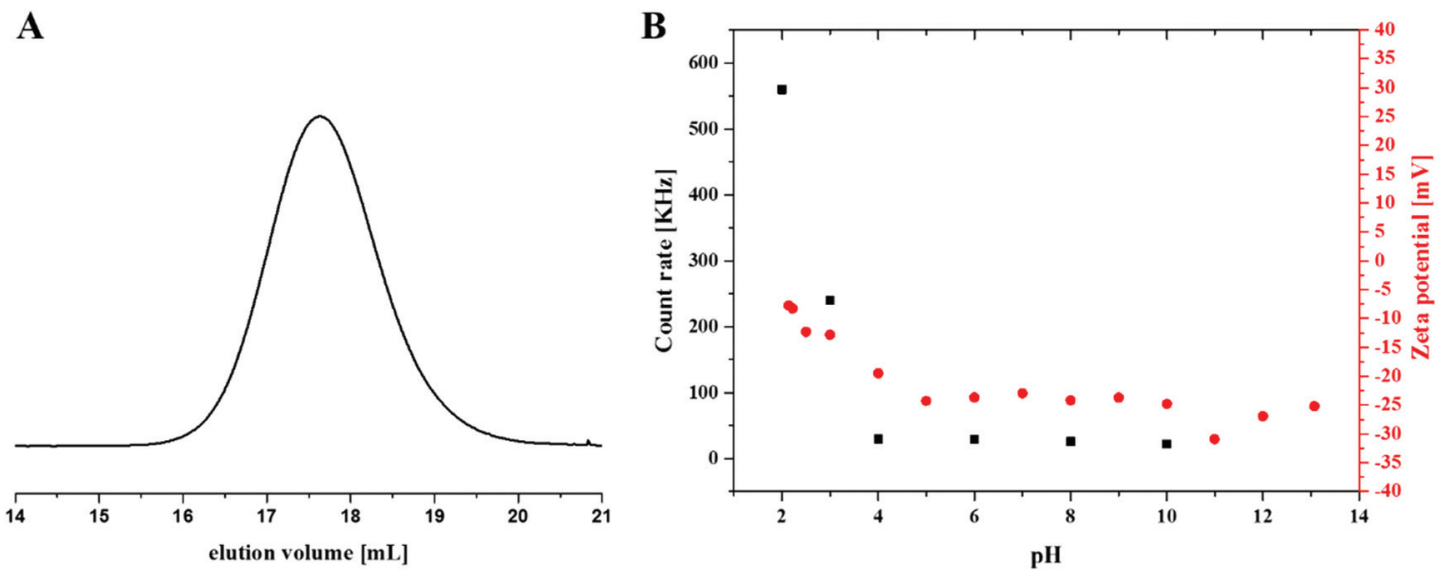

Fig. 3 (A) SEC elution trace of $\mathrm{PAA}_{25}-b-\mathrm{PDha}_{50}$ in $0.1 \mathrm{M} \mathrm{Na}_{2} \mathrm{HPO}_{4}$ at $\mathrm{pH} 9$ (Pullulan calibration); (B) zeta potential measurements of PAA $25-b$ $\mathrm{PDh}_{50}$ at different $\mathrm{pH}$ values (red squares), and the corresponding average count rates of $\mathrm{PAA}_{25}-b-\mathrm{PDha}_{50}$ in aqueous media at different $\mathrm{pH}$ value as obtained using DLS experiments (black squares).

distribution, indicating an intact polymer backbone after the different reaction steps (Table 1, Fig. 3A).

\section{Synthesis of $\mathrm{PS}_{30}-b-\mathrm{PDha}_{40}$}

$\mathrm{PS}_{30}-b$-PtBAMA 40 exhibits and 2-1.5 ppm) and signals for PS $(6.5-7.5 \mathrm{ppm}$, PtBAMA $\quad(5.7-5.2 \quad \mathrm{ppm} \quad-\mathrm{NH}$,
3.45-3.89 ppm, $-\mathrm{OCH}_{3}, 3.3-2 \mathrm{ppm}-\mathrm{C}-\mathrm{CH}_{2}-$, 1.6-1.3 ppm -(C$\left.\left(\mathrm{CH}_{3}\right)_{3}\right)$, Fig. S5 $\dagger$ ). In ${ }^{13} \mathrm{C}-\mathrm{NMR}$, signals for PS $(147,129,127,61$ and $46 \mathrm{pm})$ as well as for PtBAMA $(174,156,81,61,55,42$ and $30 \mathrm{ppm}$ ) can be observed (Fig. S6†). Deprotection of the Boc group was carried out using 10 eq. TFA in dichloromethane at RT. After $19 \mathrm{~h}$, the reaction mixture was neutralized with a 
sodium hydroxide solution and the block copolymer was precipitated in hexane. According to ${ }^{1} \mathrm{H}-\mathrm{NMR}$ in d-TFA, a deprotection of $93 \%$ could be confirmed through the decrease of the signal at $1.5 \mathrm{ppm}$. Besides, the corresponding signal of the amine function changed from $5.5 \mathrm{ppm}$ in $\mathrm{CDCl}_{3}$ to $3.0 \mathrm{ppm}$ in $d$-TFA, similar to earlier observations for PnBA- $b$-PAMA. Also, here this treatment leads to a simultaneous removal of the methyl ester (4.2 ppm, Fig. S5 $\dagger$ ) of approximately 75\% $\left(\mathrm{PS}_{30}-b\right.$ $\left(\mathrm{PAMA}_{10}\right.$-Co-PDha $\left.{ }_{30}\right)$ ). In ${ }^{13} \mathrm{C}-\mathrm{NMR}$ (d-TFA), the backbone carbon signals (59 and $39 \mathrm{ppm}$ ) and the signal for the methyl ester (50 ppm) could be detected (Fig. S8 $\dagger$ ).

Removal of the methyl ester was performed as described above for $\mathrm{P} n \mathrm{BA}_{25}-b$-PtBAMA 50 using 21 eq. $\mathrm{LiOH} \cdot \mathrm{H}_{2} \mathrm{O}$ for $24 \mathrm{~h}$ at $100{ }^{\circ} \mathrm{C}$. After neutralization and precipitation, the resulting block copolymer was characterized by NMR in DMSO- $d_{6}$. Here, only signals for the PS segment (7.3-6.3 ppm) and the block copolymer backbone (2.4-1.32 ppm) were observed, and the intensity of the Boc group decreased to $7 \%$ (1.25 ppm). However, almost complete cleavage of the methyl ester was achieved ( $\left.>90 \%, \mathrm{PS}_{30}-b-\left(\mathrm{P}_{\mathrm{BAMA}}-\mathrm{Co}-\mathrm{PDha}_{37}\right)\right)$. In this particular case, solid state NMR was carried out due to low solubility of the material in common solvents $\left({ }^{1} \mathrm{H}-\mathrm{NMR}\right.$ : Fig. S7, $\dagger$ ${ }^{13} \mathrm{C}-\mathrm{NMR}$ : Fig. S8 $\dagger$ ). Here, the carbons of the aromatic ring $(146,129 \mathrm{ppm})$ can be detected (Fig. S7 $\dagger$ ), as well as the carbonyl moiety of the ester (179 ppm) and the Boc group (157 ppm). Further, a small signal for the Boc group was detected at $30 \mathrm{ppm}$, again confirming the partial cleavage, and additionally the backbone signals (62 and $46 \mathrm{ppm}$ ) were visible.

The preparation of $\mathrm{PS}_{30}-b-\mathrm{PDha}_{40}$ was carried out from $\mathrm{PS}_{30}-b$-(PtBAMA $3-c o$-PDha $\left.{ }_{37}\right)$ by hydrolysis with TFA as described above. After neutralization and dialysis against water, the block copolymer was characterized by ${ }^{1} \mathrm{H}-\mathrm{NMR}$ in DMSO. Signals for the PS segment at 7.2-6.4 ppm and the block copolymer backbone (2.2-1.5 ppm) were observed, as well as a small amount of remaining Boc group (7\%, 1.5 ppm, Fig. S7 $\dagger$ ). Solid state ${ }^{13} \mathrm{C}-\mathrm{NMR}$ of $\mathrm{PS}_{40}-b-\mathrm{PDha}_{30}$ revealed signals at 180, 146, 62 and 46 and 42 ppm (Fig. S8†).

\section{Solution behavior of $\mathrm{PAA}_{25}-b-\mathrm{PDha}_{50}$ and $\mathrm{PS}_{30}-b-\mathrm{PDha}_{40}$}

We were interested in the solution behavior of the above described amphiphilic and double hydrophilic block copolymers. Starting with $\mathrm{PAA}_{25}-b$-PDha ${ }_{50}$, we investigated the $\mathrm{pH}$ dependent solubility and net charge using a combination of zeta-potential measurements and dynamic light scattering (Fig. 3B). Starting under basic conditions ( $\mathrm{pH} 14)$, the block copolymer shows a negative zeta potential (approximately $-25 \mathrm{mV}$ ) and this value remains more or less constant until $\mathrm{pH} 5$, presumably due to the presence of deprotonated $-\mathrm{COO}^{-}$ moieties. Upon acidification, at $\mathrm{pH} 4$ the zeta potential starts to increase up to $-5 \mathrm{mV}$ at $\mathrm{pH}$. This hints towards both the protonation of the carboxylates as well as the amino moiety. We explain the fact that the zeta potential remains slightly negative mainly by partial aggregation of the block copolymer at lower $\mathrm{pH}$ values.
These findings were supported by DLS experiments at different $\mathrm{pH}$ values in aqueous solution at a concentration of $1 \mathrm{~g} \mathrm{~L}^{-1}$ (Fig. 3B and 4). As can be seen, below $\mathrm{pH}$ values of 4 , a significant increase in apparent count rate occurs, hinting towards aggregation of $\mathrm{PAA}_{25}-b$-PDha ${ }_{50}$. This process is accompanied by increasing turbidity. DLS CONTIN plots of $\mathrm{PAA}_{25}-b$-PDha 50 at different $\mathrm{pH}$ values show mainly unimers at pH 10, as well as at pH 6 and 4 (Fig. 4, hydrodynamic radii of 1.5 to $3 \mathrm{~nm}$ are detected, hinting towards the presence of mainly block copolymer unimers). Under acidic conditions ( $\mathrm{pH}$ 3), distinct differences were observed as the degree of protonation for both the carboxylic acid groups of PAA and PDha increases. This leads to a decreased solubility, followed by aggregation and the respective DLS experiments revealed an apparent hydrodynamic radius of $220 \mathrm{~nm}$, pointing towards the presence of loosely defined block copolymer aggregates (Fig. 4).

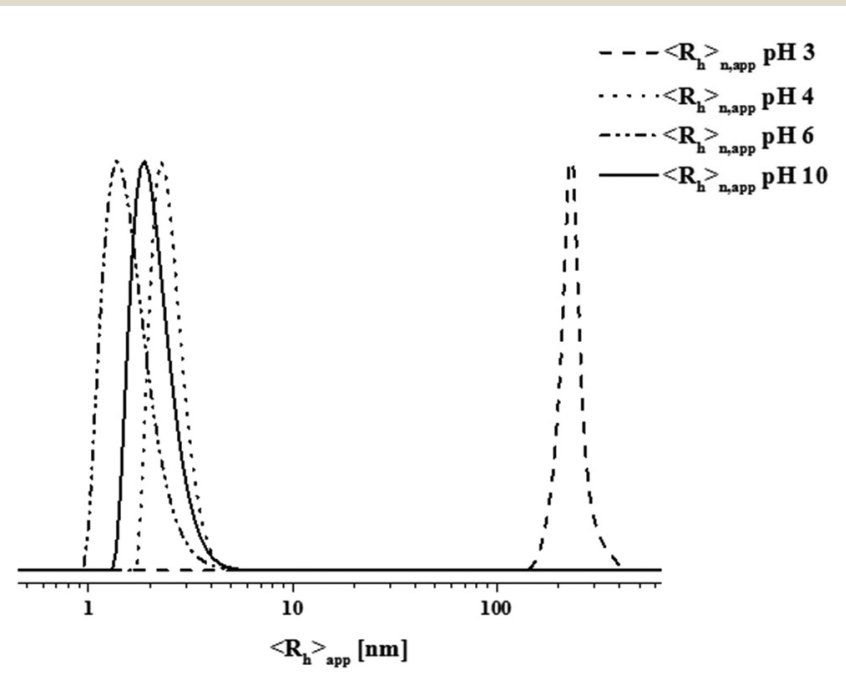

Fig. 4 DLS CONTIN plots of $\mathrm{PAA}_{25}-b-\mathrm{PDha}_{50}$ at $\mathrm{pH} 10$ (solid black line, $\left\langle R_{\mathrm{h}}\right\rangle_{n}$, app $=2 \mathrm{~nm}$ ), pH 6 (dashed-dotted line, $\left\langle R_{\mathrm{h}}\right\rangle_{n, \text { app }}=1.5 \mathrm{~nm}$ ), $\mathrm{pH} 4$ (dotted line, $\left\langle R_{\mathrm{h}}\right\rangle_{n}$, app $=2.5 \mathrm{~nm}$ ), and $\mathrm{pH} 3$ (dashed line, $\left\langle R_{\mathrm{h}}\right\rangle_{n}$, app $=$ $220 \mathrm{~nm}$ ) - concentration was $1 \mathrm{~g} \mathrm{~L}^{-1}$ in all cases.

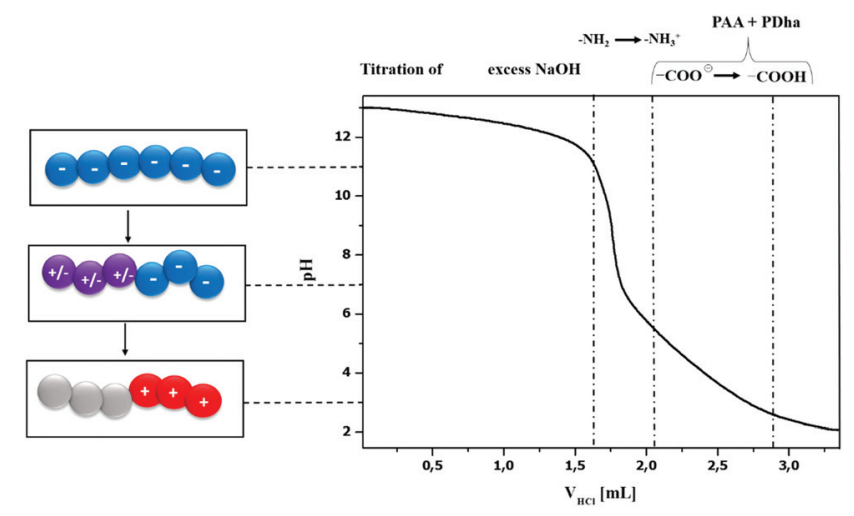

Fig. 5 Potentiometric titration of $\mathrm{PAA}_{25}-b-\mathrm{PDha}_{50}$ with $0.1 \mathrm{M} \mathrm{HCl}$ starting from $0.1 \mathrm{M} \mathrm{NaOH}$. 
A

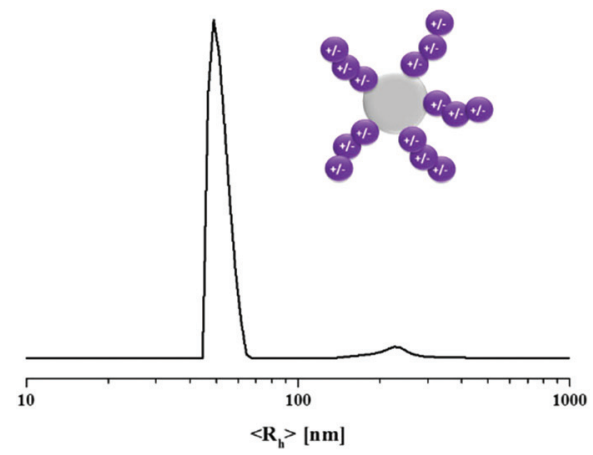

B

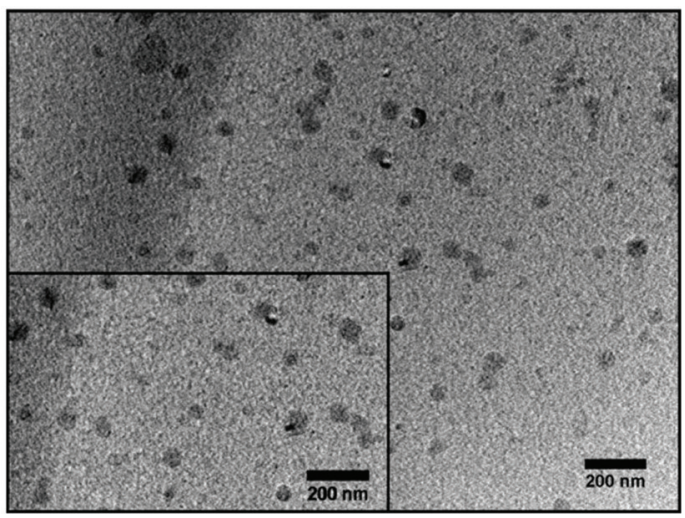

Fig. 6 DLS CONTIN plot of $\mathrm{PS}_{30}-b-\mathrm{PDha}_{40}$ in $\mathrm{D}_{2} \mathrm{O}\left(0.1 \mathrm{~g} \mathrm{~L}^{-1},<R_{\mathrm{h}}\right\rangle_{n}$, app $=51,224 \mathrm{~nm}$, (A) and cryo-TEM micrograph of a micellar solution of PS $30-b-$ $\mathrm{PDha}_{40}$ (B).

We also carried out potentiometric titrations of $\mathrm{PAA}_{25}-b$ PDha $_{50}$ at a concentration of $3 \mathrm{~g} \mathrm{~L}^{-1}$, starting under basic conditions ( $\mathrm{pH}$ 13, $0.1 \mathrm{M} \mathrm{NaOH}$, double negatively charged block copolymer unimers are present as discussed above) by stepwise addition of $\mathrm{HCl}$ (0.1 M, Fig. 5). Here, the first steps ( $\mathrm{pH}$ 13.0-11.0) mainly correspond to the neutralization of excess $\mathrm{NaOH}$. According to previous studies, the $\mathrm{p} K_{\mathrm{B}}$ of the $-\mathrm{NH}_{2}$ moiety in PDha is around 9.2, indicating a pH-range of increasing protonation from $\mathrm{pH} 11-7 .{ }^{29}$ In that region, the block copolymer consists of a negatively charged segment (PAA) and a zwitterionic block (PDha). Further decrease of the $\mathrm{pH}$ value leads to continuous protonation of the amino functionality. For the - $\mathrm{COOH}$ groups, typical $\mathrm{p} K_{\mathrm{A}}$ values are reported around $6-7 .^{40-44}$ Therefore, at a $\mathrm{pH}$ value of 5 the majority of $-\mathrm{COOH}$ moieties is protonated, leading to a charge neutral PAA segment and mainly positive charges within the PDha block. This then leads to aggregation at lower $\mathrm{pH}$ values $(\mathrm{pH}<4)$, accompanied by increasing turbidity and precipitation at $\mathrm{pH} 2.3$ (see also DLS experiments).

We were also interested in the solution properties of amphiphilic $\mathrm{PS}_{30}-b$-PDha 40 and therefore dissolved the block copolymer in water $\left(\mathrm{D}_{2} \mathrm{O}\right)$ by short heating to $80{ }^{\circ} \mathrm{C}$ at a concentration of $c=0.1 \mathrm{~g} \mathrm{~L}^{-1}$. After cooling down, a turbid solution was obtained, which we ascribed to the micellization of $\mathrm{PS}_{30}-b$ PDha $_{40}$ (Fig. 6). The core of these aggregates is formed by the hydrophobic PS block and the corona is formed by zwitterionic PDha under these conditions. Subsequent DLS measurements revealed the presence of micelles with a hydrodynamic radius of $51 \mathrm{~nm}$, as well as some larger aggregates above $200 \mathrm{~nm}$ in radius (Fig. 6A). Additional cryo-TEM measurements revealed the presence of aggregates with a diameter of $67 \mathrm{~nm} \pm 10 \mathrm{~nm}$ (measurement of 50 particles), being in relatively good agreement with the size regime obtained from DLS experiments as here only the PS core is directly visible (Fig. 6B).

\section{Conclusion}

We have demonstrated the transformation of previously synthesized PS- $b$-PtBAMA and PnBA- $b$-PtBAMA diblock copolymers into double hydrophilic PAA- $b$-PDha and amphiphilic PS- $b$ PDha materials by sequential deprotection steps. Thereby, we discovered that neither the TFA-mediated deprotection of the Boc moiety nor the hydrolysis of the methyl ester under basic conditions using $\mathrm{LiOH}$ are truly selective. Instead, in both cases already considerable amounts of PDha are generated directly - as evidenced mainly by in-depth NMR investigations. Although the focus of this work here was mainly put on the final PDha-based block copolymers, future work will also be directed towards different monomer substitution patterns which can be orthogonally addressed. Nevertheless, the resulting block copolymers revealed interesting solution properties in aqueous media, as shown for example by $\mathrm{pH}$-dependent DLS and zeta potential measurements in the case of PAA- $b$ PDha or first micellization studies of PS- $b$-PDha. Such materials in our opinion are of interest as building blocks for micellar interpolyelectrolyte complexes (IPECs) or for the preparation of membranes with charge-tunable separation layers.

\section{Acknowledgements}

The authors thank Kristin Schreyer (FSU Jena) for help with SEC measurements and Philip Biehl for performing cryo-TEM. FHS is grateful to the Thuringian Ministry of Science, Education, and Culture (TMBWK; grants \#B515-10065, ChaPoNano and \#B514-09051, NanoConSens). We would further like to acknowledge the NMR-platform at the FriedrichSchiller-University Jena for support in NMR spectroscopy. FHS is also grateful to Ulrich S. Schubert for continuous support.

\section{References}

1 A. V. Dobrynin and M. Rubinstein, Prog. Polym. Sci., 2005, 30, 1049-1118.

2 A. Laschewsky, Curr. Opin. Colloid Interface Sci., 2012, 17, 56-63. 
3 D. V. Pergushov, A. H. E. Müller and F. H. Schacher, Chem. Soc. Rev., 2012, 41, 6888-6901.

4 A. V. Dobrynin, R. H. Colby and M. Rubinstein, J. Polym. Sci., Part B: Polym. Phys., 2004, 42, 3513-3538.

5 A. B. Lowe and C. L. McCormick, Chem. Rev., 2002, 102, 4177-4190.

6 A. Laschewsky, Polymers, 2014, 6, 1544.

7 S. Kudaibergenov, W. Jaeger and A. Laschewsky, in Supramolecular Polymers Polymeric Betains Oligomers, Springer Berlin Heidelberg, Berlin, Heidelberg, 2006, pp. 157-224.

8 I. Roy and M. N. Gupta, Chem. Biol., 2003, 10, 1161-1171.

9 Y. Qiu and K. Park, Adv. Drug Delivery Rev., 2001, 53, 321339.

10 D. A. Mortimer, Polym. Int., 1991, 25, 29-41.

11 S. Jiang and Z. Cao, Adv. Mater., 2010, 22, 920-932.

12 F. Xuan and J. Liu, Polym. Int., 2009, 58, 1350-1361.

13 P.-S. Liu, Q. Chen, S.-S. Wu, J. Shen and S.-C. Lin, J. Membr. Sci., 2010, 350, 387-394.

14 A. B. Ihsan, T. L. Sun, T. Kurokawa, S. N. Karobi, T. Nakajima, T. Nonoyama, C. K. Roy, F. Luo and J. P. Gong, Macromolecules, 2016, 49, 4245-4252.

15 J. Zhao, N. A. D. Burke and H. D. H. Stöver, RSC Adv., 2016, 6, 41522-41531.

16 X. Shen, X. Yin, Y. Zhao and L. Chen, Colloid Polym. Sci., 2015, 293, 1205-1213.

17 Y. K. Jhon, S. Arifuzzaman, A. E. Özçam, D. J. Kiserow and J. Genzer, Langmuir, 2012, 28, 872-882.

18 Q. Zhang and R. Hoogenboom, Chem. Commun., 2015, 51, 70-73.

19 R. Wang and A. B. Lowe, J. Polym. Sci., Part A: Polym. Chem., 2007, 45, 2468-2483.

20 M. Kamachi, M. Kurihara and J. K. Stille, Macromolecules, 1972, 5, 161-167.

21 E. Giebeler and R. Stadler, Macromol. Chem. Phys., 1997, 198, 3815-3825.

22 E. Betthausen, M. Drechsler, M. Förtsch, F. H. Schacher and A. H. E. Müller, Soft Matter, 2011, 7, 8880-8891.

23 F. Schacher, A. Walther and A. H. E. Müller, Langmuir, 2009, 25, 10962-10969.

24 E. Betthausen, M. Drechsler, M. Förtsch, D. V. Pergushov, F. H. Schacher and A. H. E. Müller, Soft Matter, 2012, 8, 10167-10177.

25 A. C. Rinkenauer, A. Schallon, U. Günther, M. Wagner, E. Betthausen, U. S. Schubert and F. H. Schacher, ACS Nano, 2013, 7, 9621-9631.
26 Z. A. Jiménez and R. Yoshida, Macromolecules, 2015, 48, 4599-4606.

27 I. Javakhishvili, K. Jankova and S. Hvilsted, Polym. Chem., 2013, 4, 662-668.

28 M. Billing and F. H. Schacher, Macromolecules, 2016, 49, 3696-3705.

29 U. Günther, L. V. Sigolaeva, D. V. Pergushov and F. H. Schacher, Macromol. Chem. Phys., 2013, 214, 22022212.

30 M. von der Lühe, U. Günther, A. Weidner, C. Gräfe, J. H. Clement, S. Dutz and F. H. Schacher, RSC Adv., 2015, 5, 31920-31929.

31 R. E. Hermes, L. J. Mathias and J. W. Virden, Macromolecules, 1987, 20, 901-903.

32 L. J. Mathias and R. E. Hermes, Macromolecules, 1988, 21, 11-13.

33 L. J. Mathias and R. E. Hermes, Macromolecules, 1986, 19, 1536-1542.

34 C. R. Morcombe and K. W. Zilm, J. Magn. Reson., 2003, 162, 479-486.

35 P. Leroy, C. Tournassat, O. Bernard, N. Devau and M. Azaroual, J. Colloid Interface Sci., 2015, 451, 21-39.

36 A. M. Walji, E. D. Hostetler, H. Selnick, Z. Zeng, P. Miller, I. Bennacef, C. Salinas, B. Connolly, L. Gantert, M. Holahan, S. O’Malley, M. Purcell, K. Riffel, J. Li, J. Balsells, J. A. Obrien, S. Melquist, A. Soriano, X. Zhang, A. Ogawa, S. Xu, E. Joshi, J. Della Rocca, F. J. Hess, J. Schachter, D. Hesk, D. Schenk, A. Struyk, K. Babaoglu, T. G. Lohith, Y. Wang, K. Yang, J. Fu, J. L. Evelhoch and P. J. Coleman, J. Med. Chem., 2016, 59, 4778-4789.

37 T. Zhou, R. C. Hider, P. Jenner, B. Campbell, C. J. Hobbs, S. Rose, M. Jairaj, K. A. Tayarani-Binazir and A. Syme, Eur. J. Med. Chem., 2010, 45, 4035-4042.

38 J. Wang, Y.-L. Liang and J. Qu, Chem. Commun., 2009, 5144-5146.

39 H. Gunaydin and K. N. Houk, J. Am. Chem. Soc., 2008, 130, 15232-15233.

40 B. N. Dickhaus and R. Priefer, Colloids Surf., A, 2016, 488, 15-19.

41 W. Guo and N. Hu, Biophys. Chem., 2007, 129, 163-171.

42 M.-K. Chun, C.-S. Cho and H.-K. Choi, J. Controlled Release, 2002, 81, 327-334.

43 M. Guo, Y. Yan, H. Zhang, H. Yan, Y. Cao, K. Liu, S. Wan, J. Huang and W. Yue, J. Mater. Chem., 2008, 18, 5104-5112.

44 R. Dong, M. Lindau and C. K. Ober, Langmuir, 2009, 25, 4774-4779. 